\title{
Evaluación del proceso de clasificación de café (Coffee arabica L.) por el método de la espectroscopia infrarroja FTIR
}

\section{Evaluation of the coffee classification process (Coffee arabica L.) by the FTIR infrared spectroscopy method}

\author{
Christian Andrés Manrique Cortés ${ }^{[1]}$ y Nelson Gutiérrez Guzmán ${ }^{[2]}$
}

\section{Resumen}

El objetivo de este trabajo fue evaluar la diferencia de los granos de cafés sanos y los granos de cafés defectuosos (Negros, Vinagre Blanqueado, Vinagre Color y Broca), los cuales tienen una alta incidencia negativa en el momento del análisis sensorial. Se examinó el café por el método de la espectroscopia infrarroja FTIR generando unas curvas características en un espectro de onda cuyo rango va de los 750 a los $4000 \mathrm{~cm}^{-1}$. Esto puede ser una herramienta muy valiosa al momento de hacer una identificación previa a la catación para determinar si el café tiene algún defecto en taza que pueda atentar contra su calidad. Mediante los análisis realizados con el equipo Agilent Cary 630 ATR- FTIR analizador se puede llegar a desarrollar una metodología rápida y confiable para la identificación entre cafés limpios y cafés defectuosos.

Palabras clave: Espectroscopia infrarroja FTIR, Análisis sensorial del café.

\section{Abstract}

The objective of this work was to evaluate the differentiation of healthy coffee beans and defective coffee beans (Black, Bleached Vinegar, Vinegar Color and Broca), which have a high negative impact on the sensory moment. The coffee was examined by the FTIR infrared spectroscopy method, generating characteristic curves in a wave spectrum ranging from 750 to $4000 \mathrm{~cm}-1$. This can be a very valuable tool at the time of making a prior identification. Through the analysis carried out with the Agilent Cary 630 ATR-FTIR analyzer, it can be developed quickly and reliably for the identification between clean coffee and defective coffee.

Keywords: FTIR spectrophotometer, Sensory analysis of coffee.

\footnotetext{
1. Ingeniero Agrícola. Federación Nacional de Cafeteros. Avenida Pastrana, Cra 1. Neiva-Huila. mancrist@gmail.com

2. PhD. Docente. Universidad Surcolombiana. Avenida Pastrana, Cra 1. Neiva-Huila.ngutierrezg@usco.edu.co
} 


\section{Introducción}

El sabor y aromas particulares de la bebida de café son el resultado de alrededor de mil sustancias químicas que se origina en la semilla de café y se preparan para la apreciación sensorial en el momento del consumo de dicho grano (Puerta, Q., 1993). Las cualidades organolépticas o sensoriales del café comprenden el aroma, la acidez, el amargor, el cuerpo, el sabor y la impresión global de la bebida. Por su intensidad y balance se mide la calidad del café (Clarke, K., 1985). El café colombiano se cataloga como suave lavado, debido a que se obtiene de variedades de la especie Coffea arabica, cafés arábigos, y se procesa por vía húmeda en su beneficio. El proceso por vía húmeda comprende las siguientes etapas: recolección del café en cereza, despulpado, fermentación, lavado y secado hasta obtener café pergamino seco, que luego se trilla para producir café almendra (Green coffee) para exportación (Arcila, J.; et al., 2007). El proceso vía húmeda de café maduro sano y el control de las condiciones y equipos en cada etapa del beneficio permite obtener la mejor calidad de café y gracias a ello se le ha otorgado la denominación de origen al café del Huila (Federación de Cafeteros, 2015).

El mercado internacional del café ha cambiado dramáticamente en los últimos años, pues aumentó la producción potencial de los países productores con un lento crecimiento en el consumo de la bebida per cápita. Igualmente emergieron mezclas que utilizan cafés de menos calidad, existe una mayor concentración en el final de la cadena productiva y surgieron nuevos nichos de mercado, como los cafés especiales en sus distintas formas, que valoran de manera particular ciertas características del producto. Dichos cambios trajeron consigo importantes desafíos a la caficultura del país, frente a los cuales se requieren acciones para mejorar el nivel de competitividad de los productores. En el departamento del Huila, al ser el primer productor de café a nivel nacional con una participación del $18.48 \%$ lo cual equivale a 800 mil sacos de 60 kilos de café pergamino seco se debe tener un especial cuidado para conservar la calidad. Siempre en busca de la mejora de los procesos se realizó las pruebas experimentales mediante la espectroscopia infrarroja FTIR que es el método utilizado para determinar las estructuras de moléculas con características de absorción de la radiación infrarroja según su vibración molecular. La región Infrarrojo o IR va de 12.800 a $10 \mathrm{~cm}^{-1}$ y se puede dividir en la región de IR cercano (NIR) (12.800-4000 cm $\mathrm{cm}^{-1}$ ), IR medio (4.000-400 $\left.\mathrm{cm}^{-1}\right)$ e IR lejano o FAR-IR (50-400 $\left.\mathrm{cm}^{-1}\right)$. El espectro vibracional de una molécula se considera una propiedad física única y por tanto característica de ésta molécula. Así, entre otras aplicaciones, el espectro IR se puede usar como "huella dactilar" en la identificación de muestras de lotes de café mediante la comparación con espectros de referencia (Laboratorio de Técnicas Instrumentales, 2018).

\section{Metodología}

\subsection{Evaluación de los granos de café excelso utilizando la metodología de espectro infrarrojo FTIR}

Se seleccionan 5 muestras de pasillas cada una de 20 gramos (gr) como se evidencia en la figura 1.
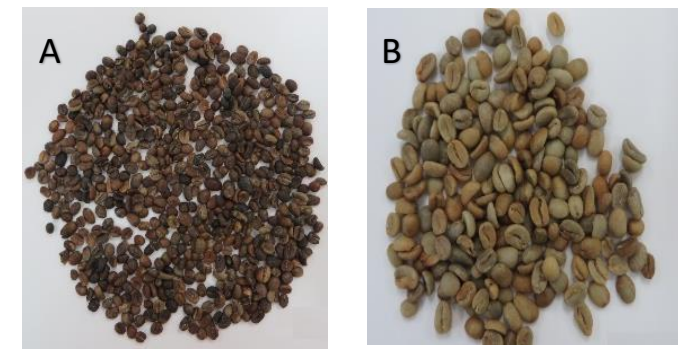

Figura 1. Muestras de pasillas. A) Negros; B
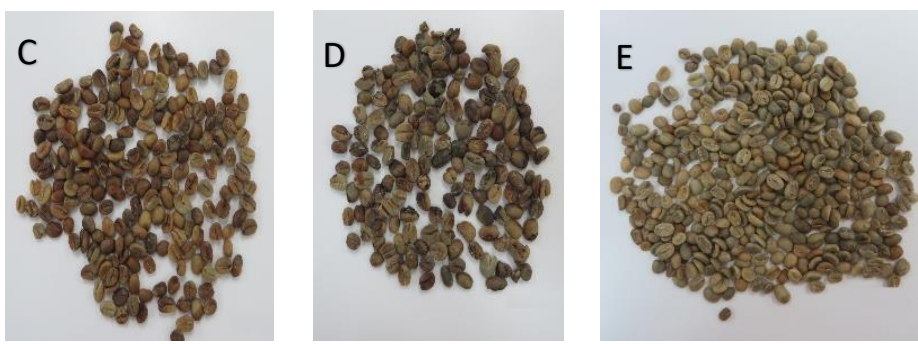

B) Vinagres Blancos; C Recuperable 
Las muestras evaluadas mediante el método FTIR se utilizó café excelso molido lo cual mejora la fiabilidad de respuesta del equipo, usando una pequeña cantidad en el lente reflectante de cristal de diamante del equipo Agilent Cary 630 ATR- FTIR analizador figura 2. Las muestras se presionan sobre el cristal de diamante el cual es el encargado de tomar las lecturas, los cuales arrojaron los resultados en un tiempo de 30 segundos evidenciando los espectros infrarrojos de la muestra.

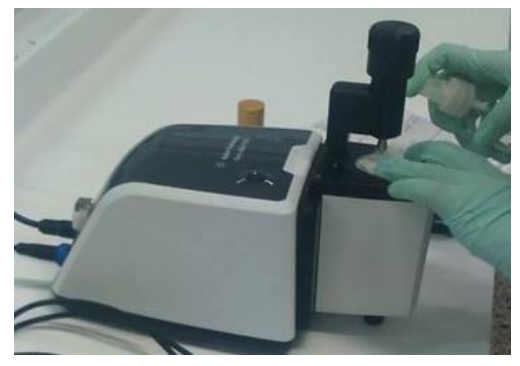

Figura 2. Equipo Agilent Cary 630 ATR- FTIR

\section{Resultados}

\subsection{Evaluación de los granos de café excelso utilizando la metodología de espectro infrarrojo FTIR}

El espectro típico de FTIR obtenidos con para muestras de café verde (Excelso) son evidenciados en la figura 3, la longitud de onda analizada corresponde al intervalo 750-4000 $\mathrm{cm}^{-1}$, los resultados obtenidos en este análisis preliminar, permiten afirmar que la huella digital del café en diferentes variedades mantiene constantes sus características. Una asignación completa de las bandas espectrales es bastante difícil, y no es el alcance de este trabajo. Además, los datos de la literatura sobre FTIR del café sólo están disponibles para muestras de café tostado, por lo que una comparación directa no se puede hacer. No obstante, se pueden discutir algunos aspectos cualitativos de los espectros.

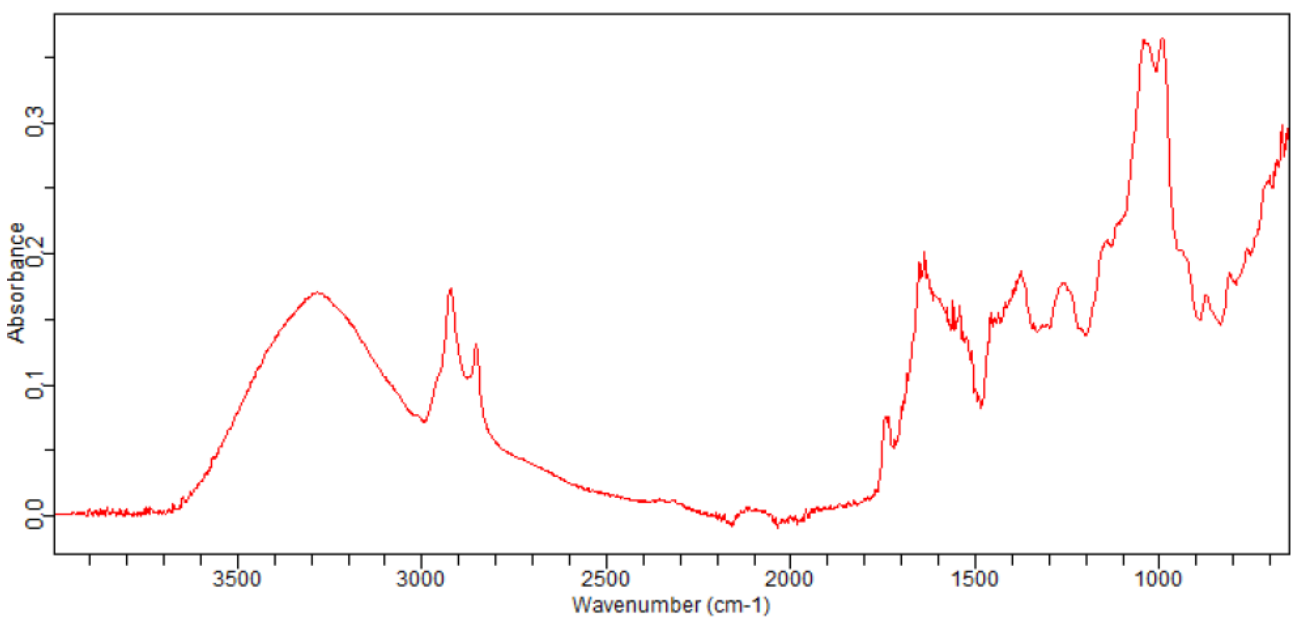

Figura 3. Curva típica de grano de café excelso en espectros FTIR

Los espectros obtenidos por la transmisión y reflectancia son similares debido a que las bandas más importantes se pueden ver los mismos tipos de espectro. También, intensidad mayor de los picos pueden ser observadas en los espectros que empleaba $\mathrm{KBr}$ (transmisión y reflectancia difusa, Figura 3), en el 1800-800 $\mathrm{cm}^{-1}$ 1,5 rango. Las dos bandas nítidas que se pueden ver en el rango 3.000-2.800 $\mathrm{cm}^{-1}$ en las muestras de café tanto Arábica y Robusta 
tostado, pero no se intentó identificación (Kemsley et al., 1995). No obstante, los estudios de análisis FTIR de refrescos que contienen cafeína tienen dos picos agudos en 2882 y $2829 \mathrm{~cm}^{-1}$, con el posterior que se está correlacionado con la asimétrica estiramiento de $\mathrm{C}-\mathrm{H}$ lazos de metilo $\left(-\mathrm{CH}_{3}\right)$ del grupo en la molécula de la cafeína y la región pico siendo utilizado con éxito para desarrollar modelos predictivos para el análisis cuantitativo de cafeína (Paradkar \& Irudayaraj, 2002).

La banda aguda en $1743 \mathrm{~cm}^{-1}$ también se ha observado en Los estudios de FTIR de café tostado (Kemsley et al., 1995; Lyman et al., 2.003; Wang et al., 2009). Kemsley et al., (1995) reportaron que una banda en $1744 \mathrm{~cm}^{-1}$ fue mayor en Arábica en comparación con una muestra de café Robusta y lo atribuyó al carbonilo $(\mathrm{C}=\mathrm{O})$ de vibración asociado al grupo éster en los triglicéridos. El estudio realizado por Lyman et al. (2003) también se asocian las bandas en esa región a los ésteres alifáticos $\left(1755-1740 \mathrm{~cm}^{-1}\right)$. Una banda en $1658 \mathrm{~cm}^{-1}$ aparece en los espectros obtenidos por transmisión $\mathrm{KBr}$, como puede verse en la figura 3, y también es asociado a la absorción de cafeína (Lyman et al., 2003). Ribeiro et al. (2010) reportaron que números de onda en el intervalo de $1700-1600 \mathrm{~cm}^{-1}$ son altamente relacionado con los ácidos clorogénicos y concentración de cafeína en cafés.

Otras bandas que aparecen en menor intensidad se pueden ver en la gama de 1600-1000 cm-1. Según Kemsley et al. (1.995), Briandet et al. (1996), y Lyman et al. (2003), ácidos clorogénicos presente una fuerte absorción en la región de 1300-1150 $\mathrm{cm}^{-1}$. Clorogénico ácidos corresponden a una gran familia de ésteres formados entre ácido quínico y uno a cuatro residuos de ciertos ácidos trans-cinámico, más comúnmente cafeico, p-cumárico y ferúlico (Clifford, Kirkpatrick, Kuhnert, Roozendaal \& Salgado, 2008). La deformación axial C-O del ácido quínico se produce en el intervalo de desde 1.085 hasta $1050 \mathrm{~cm}^{-1}$ mientras que la deformación angular $\mathrm{O}-\mathrm{H}$ se produce entre 1,420 y $1330 \mathrm{~cm}^{-1}$ como se muestra en la figura 4 . El enlace éster C-O-C también absorbe en el $1300-1000 \mathrm{~cm}^{-1} \mathrm{rango}$ (Silverstein, Webster \& Kiemle, 2005). Por lo tanto, las bandas de 1381-1376 cm cm $^{-1} 1161-1153 \mathrm{~cm}^{-1}$ y $1053 \mathrm{~cm}^{-1}$

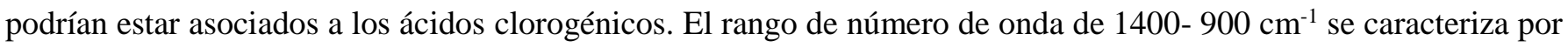
vibraciones de varios tipos de bonos, incluyendo C-H, C-O, la C-N y el P-O (Sablinskas, Steiner \& Hof, 2003; Wang et al., 2009). Los hidratos de carbono han sido previamente demostrados que presentan varias bandas de absorción en esta región (Briandet et al., 1996; Kemsley et al., 1995), lo que se espera que esta clase de compuestos también contribuirán a las varias bandas que aparecen en esta región (Craig, A.; et al., 2012).
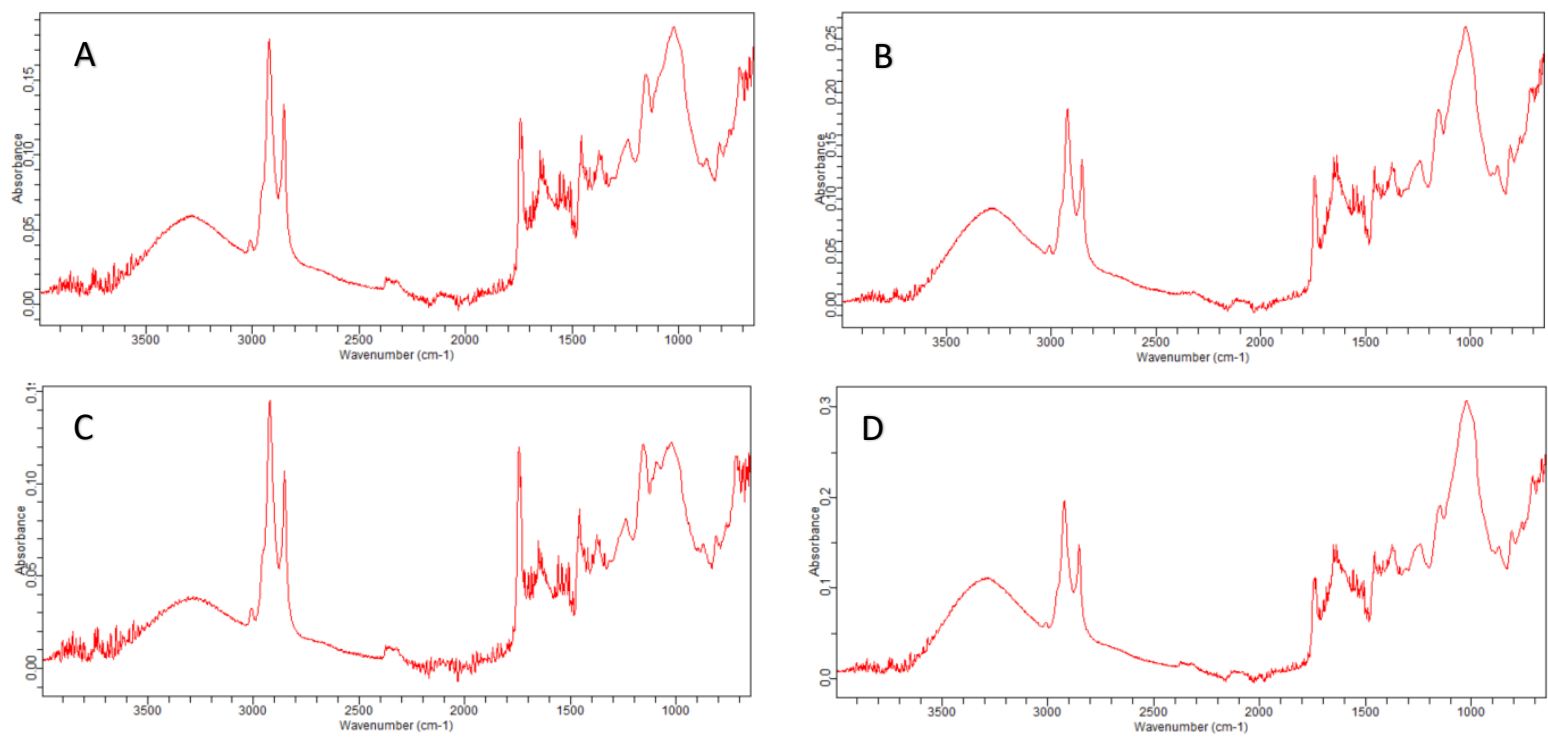

Figura 4. Curvas típicas de los principales defectos en el proceso de trilla en espectros FTIR. A) Broca; B) Negros; C) Vinagres Blanqueado; D) Vinagre Color 
Cabe señalar, que las Metodologías basadas en FTIR estén concebidas para Líquidos o Sólidos de superficies lisas grandes, haciéndolos inadecuados para su uso con granos de café enteros teniendo que moler el grano para mejores resultados. Cuando los granos se muelen, la composición química de la superficie permanecerá intacta en la muestra y por tanto lo hará contribuir a la composición de los espectros FTIR. También, todas las otras clases de compuestos son distribuidos uniformemente por todo el grano, incluyendo su superficie.

\section{Conclusión}

Los resultados basados en los espectros de reflectancia FTIR indicaron una diferencia en la longitud de onda que se expresa en el rango de los 2700 y $3000 \mathrm{~cm}^{-1}$ en los cafés excelsos y defectuosos los cuales serían de gran ayuda para una identificación previa a la catación lo cual permitiría detectar lotes con problemas de calidad. Lo cual ratifica la importancia de las pruebas FTIR para cafés excelsos y es importante a futuro ya que si se profundiza en un modelo que indique que las muestras de café excelso y pasilla podrían ser separadas en grupos distintos, basado en la transmitancia o reflectancia espectros.

\section{Referencias bibliográficas}

1. Arcila, J.; Farfán, F.; Moreno, A.; Salazar, L.; Hincapié, E., 2007. Sistemas de Producción de Café en Colombia.Fedenaricón Nacional de Cafeteros-Cenicafé.

2. Briandet, R., Kemsley, E. K., \& Wilson, R. H., 1996. Approaches to Adulteration Detection in Instant Coffees Using Infrared Spectroscopy and Chemometrics. Journal of the Science of Food and Agriculture, 71, 359$366 \mathrm{p}$.

3. Clarke, R. J.; Macrae, R., 1985. Coffee. Vol. 1. Chemistry. Essex, Elsevier Applied Science Publishers. 306 p.

4. Craig, A., Franca, A., Oliveira, L., 2012. Evaluation of the Potential of FTIR and Chemometrics for Separation Between Defective and Non-Defective Coffees. Food Chemistry. Elsevier. 1, 7 p.

5. Clifford, M. N., Kirkpatrick, J., Kuhnert, N., Roozendaal, H., \& Salgado, P. R., 2008. LC-MSn Analysis of the Cis Isomers of Chlorogenic Acids. Food Chemistry, 106, 379-385 p.

6. Federación de Cafeteros. Café del Huila con Denominación de Origen. Consultado del 20 de junio del 2015. http://www.federaciondecafeteros.org/clientes/es/buenas_noticias/cafe_del_huila_con_denominacion_de_origen/

7.Kemsley, E. K., Ruault, S., \& Wilson, R. H., 1995. Discrimination Between Coffea arabica and Coffea canephora Variant robusta Beans Using Infrared Spectroscopy. Food Chemistry, 54, 321-326 p.

8. Laboratorio de técnicas Instrumentales UVA. Consultado del 23 de noviembre del 2018. http://laboratoriotecnicasinstrumentales.es/analisis-qumicos/espectrometra-ftir

9. Lyman, D. J., Benck, R., Dell, S., Merle, S., \& Murray-Wijelath, J., 2003. FTIR-ATR Analysis of Brewed Coffee: Effect of Roasting Conditions. Journal of Agricultural and Food Chemistry, 51, 3268-3272 p.

10. Paradkar, M. M., \& Irudayaraj, J., 2002. Rapid Determination of Caffeine Content in Soft Drinks Using FTIRATR Spectroscopy. Food Chemistry, 78, 261-266p. 
11. Ribeiro, J. S., Salva, T. J., \& Ferreira, M. M., 2010. Chemometric Studies for Quality Control of Processed Brazilian Coffees Using DRIFTS. Journal of Food Quality, 33, 212-227p.

12. Sablinskas, V., Steiner, G., \& Hof, M., 2003. Applications. In G. Gauglitz \& T. Vo-Dinh (Eds.), Handbook of Spectroscopy. Weinheim, Germany: Wiley-VCH, 89, 168 p.

13. Silverstein, Webster, y Kiemle, 2005. Spectrometric Identification of Organic Compounds.

14. Wang, J., Jun, S., Bittenbender, H. C., Gautz, L., \& Li, Q. X., 2009. Fourier Transform Infrared Spectroscopy for Kona Coffee Authentication. Journal of Food Science, 74, C385-C391. 